\title{
Damping characteristics analysis on complex power grid with multi- infeed HVDC
}

\author{
Liu Zhijian, Wang Dongdong \\ Kunming university of science and technology, Yunnan,650500,China \\ wddkmust@163.com
}

\begin{abstract}
Keywords:AC-DC line; low frequency oscillation (LFO); prony algorithm; damping
ABSTRACT: In the modern electric power system,power systems expand day by day in China.More and more large capacity generators with fast control excitation systems are put into operation.Along with the introduction of the competition mechanism by power marketing,the possibility of operating at the edge of stability limit increases.The low frequency oscillation (LFO) occurs more often in the large-scale interconnected system,which threatens the safety of system operation.Furthermore, the AC/DC parallel transmission system and multi-infeed HVDC transmission system appear in several regional power systems in China, The operation conditions are more complicated,at the same time more flexible control means are brought in. Therefore, the nation-wide interconnected power systems make the LFO study in AC/DC power system necessary. The study is using of MATLAB software to build the models, based on PSS controller,which aim to study whether DC modulation effect on system low-frequency oscillation damping characteristics. And It is verified that DC modulation can improve the system damping characteristic basing on Prony algorithm. These conclusions could give some advices to damping characteristics analysis of the complex electric power system.
\end{abstract}

\section{Introduction}

Nowadays the scale of the interconnected power grid is more and more large, with the construction and development of interconnected power grid, and the low-frequency oscillation phenomenon is found in the domestic regional power grid $^{[1]}$. For southern power grid, it is a typical ultrahigh pressure, long distance, large capacity system. With the operation of ac/dc hybrid power grid, the security and stability will be complex. The dynamic stability problem since 2005 has become the leading factor power for grid safety and power limit in the main southern ${ }^{[2]}$. The main reason why the system occurs low frequency oscillationis the system of negative damping factors ${ }^{[3]}$. If the system damping is small, after the disturbance, system power oscillation is always difficult to calm which can cause continuous and low frequency oscillation ${ }^{[4]}$.Someasures of low frequency oscillation should be to enhance the system damping as a starting point, and it is effect on a radical cure of the spatial grid structure by strengthening power system low-frequency oscillation, but this investment is huge.This paper will study of ac/dc parallel transmission system in low frequency oscillation mode of nonlinear correlation function.Finally, making full use of the ac/dc parallel operation system of dc modulation effective inhibitory effect on the system low frequency oscillation to improve the low frequency oscillation damping.

\section{system description}

4 machine 11 nodes ac/dc power system structure as shown in figure 1-1, zone 1 and zone 2 communication link between the increase of a $1200 \mathrm{MW}$ unit polar dc link, rated voltage is $500 \mathrm{KV}$, rated current is $2400 \mathrm{~A}$. 


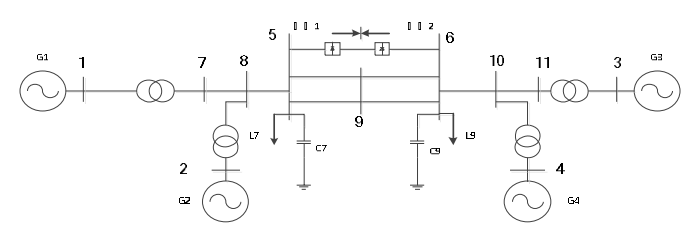

Figure 1-1 4 machine 11 nodes system structure

The length of dc link is $300 \mathrm{Km}$, the resistivity is $0.015 \Omega / \mathrm{Km}$, its total resistance is $4.5 \Omega$, inductance is $0.2376 \mathrm{H}$, the connection of each converter commutation reactance is $0.50 \Omega$, flat wave reactor used in the end of the line is $500 \mathrm{Mh}$, rectifier and inverter side all have 600 Mvar reactive power support, other parameters are as follows $K_{r} K_{t} T_{r} n_{i} a_{0} \gamma_{0} X_{d}^{\prime} T_{d 0}^{\prime} K_{U 4}=1.0, K_{t}=1.0, T_{r}=0.05, T_{i}=0.05, n_{r}=5.02, n_{i}=5.02, a_{0}=1.5, \gamma_{0}=18$. The communication system includes the use of two similar areas ofconnecting a weak link, and each area has the coupling of the two units, 1 \#, 3 \# unit of the rated capacity of 1000 MVA, 2\#,4 \# unit of the rated capacity of $5000 \mathrm{MVA}$, rated voltage is $13.8 \mathrm{KV}$, the rated capacity (MVA) and generator standard of measurement under the rated voltage $(\mathrm{kV})$ yao parameter is: $X_{d}=2.8, X_{4}=1.7, X_{d}^{\prime}=0.3, T_{d 0}^{\prime}=8.0 \mathrm{~s}, \mathrm{D}=0, H_{1}=H_{2}=4.5, H_{3}=H_{4}=5$.

Each step-up transformer impedance under 1000Mva and13.8/500kv benchmark of $0+j 0.015 \mathrm{pu}$, variable ratio of 1.0. Each generator excitation system are expressed in the first-order inertia link parameters as follows: $K_{U 1}=220, K_{U 2}=200, K_{U 3}=200, K_{U 4}=200, T_{e l}=0.5, T_{e 2}=0.5, T_{e 3}=0.05, T_{e 4}=0.05$. The rated voltage of $500 \mathrm{KV}$ power transmission system, circuit parameter in the $1200 \mathrm{Mva}$, 500KV benchmark of yao value is: $r=0.01273 \mathrm{pu} / \mathrm{km}, x_{l}=0.0009337 \mathrm{pu} / \mathrm{km}, b_{l}=0.00175 \mathrm{pu} / \mathrm{km}$. The line length is: $l_{7-8}=80 \mathrm{~km}, l_{8-5}=80 \mathrm{~km}, l_{5-9}=150 \mathrm{~km}, l_{6-9}=150 \mathrm{~km}, l_{6-10}=30 \mathrm{~km}, l_{10-11}=30 \mathrm{~km}$.

\section{system controller parameters for PSS}

Adding PSS in the above system, the purpose is to improve the system damping, suppress low frequency oscillation, so the controller parameter setting is very important, it and the controller is closely related to the damping effect of, only choose appropriate parameters can make the controller in a wider frequency range is damping effect. This article USES the method of self-excited PSS parameters design. Because self-excitation method setting process simple, and can gauge and the role of the whole system, the design method is superior.

PSS parameters should be aimed at a weak damping of electromechanical oscillation modes fo $\mathrm{r} 1 /$ setting, choose in electromechanical mode with the related units installed. Sets the transfer functi on of PSS to be/as follows: ${ }^{G \text { PSS }}(p)=\left(\frac{1+p T_{1}}{1+p T_{2}}\right)\left(\frac{p T}{1+p T}\right) K_{p s S}$ To a given $T_{2}=0.05 \sim 0.1 \mathrm{~s}, T=3 \sim 10 \mathrm{~s}$. Without PSS, $\lambda_{i}=\lambda_{i}{ }^{(0)}$;With PSS, as different $T_{1}$ makes $\lambda_{i}^{(N)}$ move in root plane, the damping ratio for the gre-atest and the basic no effect of oscillation frequency $T_{1}$ setting value for reasonable. After determining $T_{1}$, $T_{1}$ unchanged, and change the magnification factor such as PSS. Making reach the desired damping. If the resulting compared to $\lambda_{i}$ with $\lambda_{i}^{\left({ }^{(0)}\right.}$ frequency change is very small, the PSS parameter setting is co mpleted.

based on the test signal method of HVDC small signal modulator parameters setting on the sy stem damping characteristics

In ac/dc parallel systems, using and communication link parallel operation of sm-all signal mo dulation of dc transmission line can effectively suppress low frequency oscillation between the inter connected system. The commonly used types of HVDC small signal modulator for single input sing le output - lag compensation in advance.

System short circuit occurs, the corresponding bus voltage and current frequency, amplitude an d phase Angle will change, but the dc system can adopt different control modules, introduced modu lation module, the sensitivity of different modules to impr-ove the damping characteristics of the sy stem are the same, and the structure of the c-ontrol module complexity will produce great influence in the engineering application, directly or indirectly affect the power system stable operation and th e economy, relia-bility and flexibility of the project. In this paper, based on the previous theoretical 
an-alysis, alternator 1 and 3 were used respectively to Angle amplitude deviation control module an $\mathrm{d}$ a dc power amplitude deviation control module to control dc power circu-it as the offset system af ter the failure of mechanical power and electromagnetic pow-er imbalance power, in order to impro ve the damping characteristics of the system, in-hibit low-frequency oscillation of the system.

\section{the alternator Angle deviation amplitude control module into the dc modulation}

A dc link only access system, by means of ac generator 1 and 3 of the Angle dev-iation is intro duced into dc modulation amplitude control module, time characteristic of the system is back to nor mal, the introduction of dc power of the damping characte-ristics of complex systems.

Control module parameters Settings such as table 1-1:

Table 1-1 parameter Settings

Reflect the results of the system damping characteristics figure is showing in Fig 1-1

\begin{tabular}{|l|l|l|l|}
\hline frequency & damping ratio & decay factor & amplitude \\
\hline 1.130 & 0.28 & -2.053 & 585.1 \\
\hline 1.480 & 0.12 & -1.110 & 213 \\
\hline
\end{tabular}

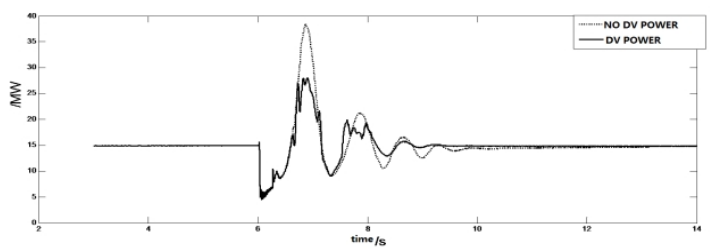

Figure 1-1 area $1 \mathrm{dc}$ power communication link before and after active power oscillation curve comparison chart adopt dc power deviation amplitude modulation control module into the dc modulation Control module parameters Settings such as table 1-2:

\begin{tabular}{|l|l|l|}
\hline Description & threshold value & gain value \\
\hline & 1.4588 & 0.79000 \\
\hline
\end{tabular}

Table 1-2 parameter Settings

Reflect the results of the system damping characteristics figure is showing in Fig 1-2:

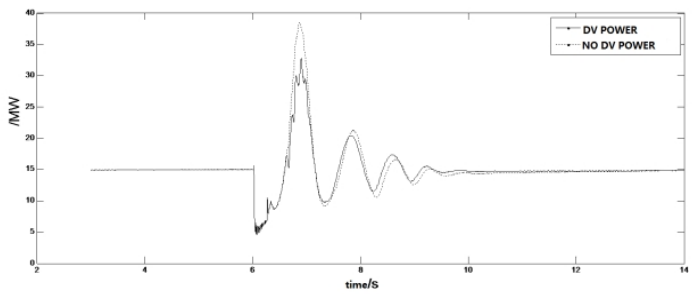

Figure 1-2 area 1 dc power communication link before and after active power oscillation curve comparison chart

\section{based on Prony analysis of dc modulation effect}

\section{Prony analysis results}

In figure 2-1,2 power oscillation curve with the Prony analysis method is analyzed, from the 7s-14s data of these two curve fitting, model 290 when fitting effect is quite good, model output and the actual system simulation data curve almost entirely coincidence. Listed in table2-1 to join dc modulation part of the index, the size of and the corresponding damping ratio;Table2-2part list by adding dc modulation index, and the corresponding damping ratio.

Damping ratio definition formula is as follows: 


$$
\zeta=\frac{-\sigma}{\sqrt{\sigma^{2}+\omega^{2}}}
$$

\begin{tabular}{|l|l|l|l|}
\hline frequency & damping ratio & decay factor & amplitude \\
\hline 1.13 & 0.286 & -2.119 & 625.4 \\
\hline 1.48 & 0.125 & -1.171 & 244.8 \\
\hline 1.97 & 0.139 & 1.415 & 70.14 \\
\hline
\end{tabular}

Table 2-1 the Prony analysis results without dc modulation

\begin{tabular}{|l|l|l|}
\hline Description & threshold value & gain value \\
\hline & 0.2 & 0.7900 \\
\hline
\end{tabular}

Table 2-2 to join dc modulation Prony analysis results

By comparing the above table 2-1 and 2-2 in the damping ratio, when the frequency of 1.130 and $1.130 \mathrm{~Hz}$, join dc modulation system damping ratio from $27.78 \%$ to $27.78 \%$ and from $11.85 \%$ to $12.52 \%$. Visible dc modulation of low frequency oscillation damping effect is obvious.

\section{Conclusion}

Aiming at analyzing complex comparatively outstanding low frequency oscillati-on of intercon nected power grid, ant then the mathematical model of ac/dc to ac/dc parallel transmission system 1 ow-frequency oscillation damping control strategy are s-tudied, based on PSS control.High efficien cy dc modulation to suppress low frequenc-y oscillation has been got to improve the dynamic stabili ty of the system, and the effe-ct is obvious. At the same time, based on Prony analysis before and aft er the introduc-tion of dc modulation system damping characteristics of the change, it is concluded $t$ -hat dc power system is introduced into really changed the total damping system, the system low fre quency oscillation suppression effect is obvious. Although studies have made some progress, but th ere are still some problems need to be further research in the future.

\section{References}

[1] DongjunYang,Jian-yongDing,HongZhou.Based on the forced oscillation theory and mechanism of low frequency oscillation parameter resonance theory analysis[J].Power System Technology,2011,3:7-12.

[2] ZhifeiLiang,KunZhang.Southern power grid frequency oscillation control strategy study[J].Automation of electric power systems,2011,35(16),54-58.

[3] DunwenSong,XuetaoYang,QiaolinDing.Large-scale interconnected power grid low-frequency oscillation analysis and control method were[J].Power grid technology,2011,35(10),22-28.

[4] ShijieHe,Kang Bo,LimingZhang. The cause of the power system low frequency oscillation and suppression method analysis[J].Power electronics,2015,1,246. 